\title{
Analysis of Water Temperature Distribution in Bathtub
}

\author{
Yanfang Wang ${ }^{1, \text { a }}$ \\ ${ }^{1}$ School of thermodynamics, North China Electric Power University, Baoding 071000, \\ China
}

a649006356@qq.com

\begin{abstract}
The article introduced a model of water temperature distribution in bathtub. The model can be used to describe the distribution of water temperature in space. Based on the heat-transfer equation while taking temperature of each point in space into account, the authors design a model that can describe the distribution of water temperature in space.
\end{abstract}

Keywords: temperature, distribution, bathtub

\section{Introduction}

In the analysis of the shape of bathtub, according to the requirements given by the problem, the authors only consider the change of temperature in height. Then, the paper simplifies the three-dimensional heat-transfer equation as the one-dimensional equation. After modeling, the authors obtain the change function of water temperature with the height of the bathtub by substituting the temperature from model one. By calculating the temperature at the bottom of the bathtub, the authors get time that we need open the faucet to maintain the water temperature.

\section{Changes of water temperature with shape}

The faucet is over the bathtub, and we assume the temperature of water surface is 40 degrees.

According to the heat-transfer equation:

$$
\rho C \frac{\partial T}{\partial t}-\lambda_{w}\left(\frac{\partial^{2} T}{\partial x^{2}}+\frac{\partial^{2} T}{\partial y^{2}}+\frac{\partial^{2} T}{\partial z^{2}}\right)=Q_{g}
$$

Due to the shape of the bathtub, we can simplify the changes of the water in the bathtub as the changes of the water in height.

So we can divide the water into certain number of layers. Thus, the temperature field in the bathtub is simplified as one-dimensional temperature condition.

We assume the bottom of the bathtub as the adiabatic boundary, so we can consider the change of water temperature as heat transfer of one-dimensional steady-state within heat sources. Then, we have the differential equations of heat transfer as follow:

$$
\frac{d^{2} T}{d x^{2}}+\frac{\phi}{\lambda_{w}}=0
$$


In the Eq.2, $x$ represents the distance from water surface to any point at the height. And we have follow boundary conditions:

$$
x=0, T=T_{s} ; x=H, \frac{d T}{d x}=0
$$

In the Eq.3, $T_{s}$ represents the temperature of water surface in the bathtub, $H$ represents the height of the bathtub.

Then, we determine the strength of virtual heat source. We can take an integral element in the height direction $d x$ as follow:

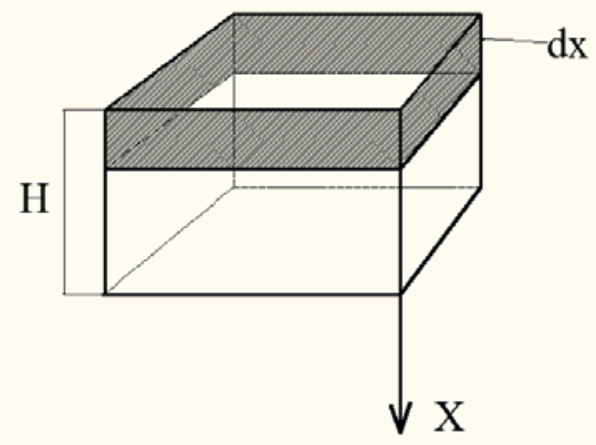

Fig.1.An integral element in the height direction of water

And we can calculate the total thermal radiation $\phi_{s}$ of the integral element $d x$ as follow:

$$
\phi_{s}=h P\left(T-T_{\infty}\right) d x
$$

The volume of the integral element is $A_{c} d x$, where $A_{c}$ represents the area of section, so we can calculate the strength of virtual heat source $\phi$ as follow:

$$
\phi=-\frac{\phi_{s}}{A_{c} d x}=\frac{h P\left(T-T_{\infty}\right)}{A_{c}}
$$

where $P$ represents section perimeter.

We can get the follow equation by substituting Eq.4 into Eq.2:

$$
\frac{d^{2} T}{d x^{2}}-\frac{h P\left(T-T_{\infty}\right)}{A_{c} \lambda_{w}}=0
$$

We can define $T-T_{\infty}$ as $\theta$, and let $m^{2}=\frac{h P}{\lambda_{w} A_{c}}$ 


$$
\frac{d^{2} \theta}{d x^{2}}-m^{2} \theta=0
$$

And we have follow boundary conditions:

$$
x=0, \theta=\theta_{0} ; x=H, \frac{d \theta}{d x}=0
$$

we can get the general solution as follow:

$$
\theta=c_{1} e^{m x}+c_{2} e^{-m x}
$$

By substituting Eq.8 into Eq.9.We calculate the result as follow:

$$
\begin{gathered}
\theta_{0}=c_{1}+c_{2} \\
c_{1} m e^{m H}+c_{2} m e^{-m H}=0 \\
\theta=\theta_{0} \frac{\cosh [m H(1-x / H)]}{\cosh (m H)}=\frac{\cosh m y}{\cosh m H}
\end{gathered}
$$

And when $x=H$, we calculate the temperature of water as follow:

$$
\theta_{H}=\theta_{0} \frac{1}{\cosh (m H)}
$$

So we can get $\theta_{0}$ by adding room temperature $T_{\infty}$ to the temperature of water surface $T$ from the model one. Then we can obtain the function of water temperature of any point in space with time by Eq.12. From this, we can get time that reheating the water to initial temperature needs.

\section{Calculation and Results}

We have basic parameters as follow:

$$
k=0.01, M_{p}=70 \mathrm{~kg}, V_{p}=0.07 \mathrm{~m}^{3}, M_{w}=230 \mathrm{~kg}, V_{w}=0.23 \mathrm{~m}^{3}, v=1 \mathrm{~m} / \mathrm{s}
$$
follow:

We can get the Fig. 1 by substituting basic parameters in Eq.13 into Eq.12 as

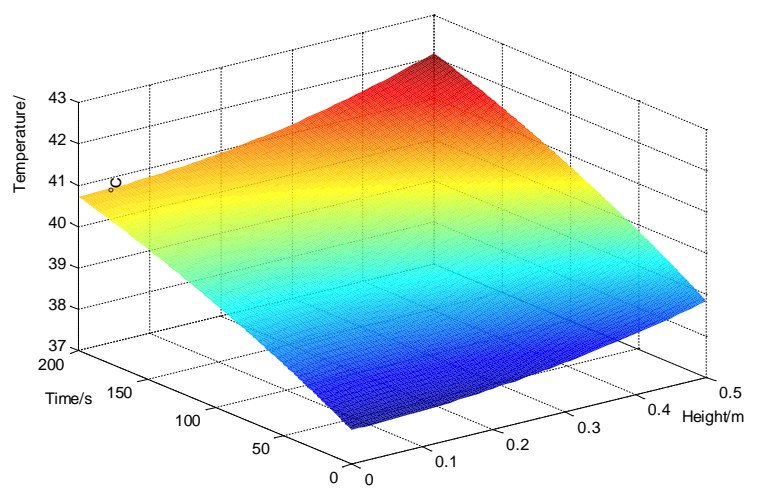

Fig.2. Distribution of water temperature in height and time with pouring hot water 


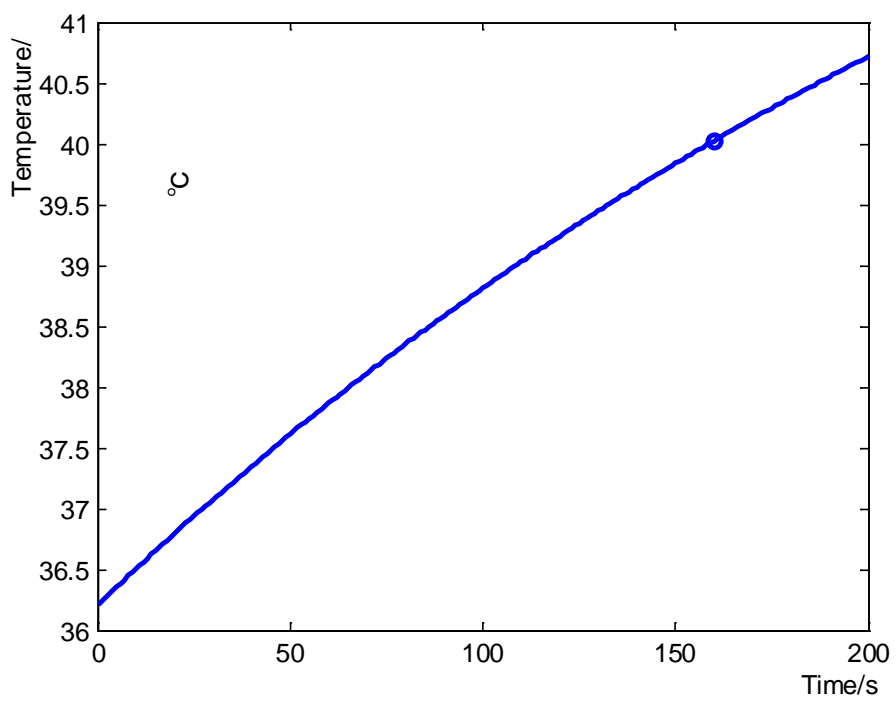

Fig.3. Curve of water temperature at the bottom with time while pouring hot water

\section{Conclusions}

In Fig.2, the red area represents the highest temperature while the blue area represents the lowest temperature. So we can find that water temperature rises with height rising when time is constant. With time of pouring hot water increasing, water temperature rises gradually at the same height.

The Fig. 3 shows that the water temperature at the bottom of tub rises with time. And after 160 seconds, water temperature is reheated to 40 degrees.

Similarly, we can obtain the curve of water temperature with change of height when temperature is 40 degrees as follow:

\section{References}

[1] Smith J M. Introduction to chemical engineering thermodynamics[D]. Rensselaer Polytechnic Institute, 1975.

[2] Prausnitz J M, Lichtenthaler R N, de Azevedo E G. Molecular thermodynamics of fluid-phase equilibria[M]. Pearson Education, 1998.

[3] Trefethen L N. Spectral methods in MATLAB[M]. Society for Industrial and Applied Mathematics, 2000.

[4] Munson B R, Young D F, Okiishi T H. Fundamentals of fluid mechanics[J]. New York, 1990, 3(4).

[5] Cathles L M. An analysis of the cooling of intrusives by ground-water convection which includes boiling[J]. Economic Geology, 1977, 72(5): 804-826. 